\title{
Laterotactile Rendering of Vector Graphics with the Stroke Pattern
}

\author{
Vincent Lévesque $^{1}$ and Vincent Hayward ${ }^{2}$ \\ 1 Department of Computer Science, University of British Columbia \\ 201-2366 Main Mall, Vancouver, BC, V6T 1Z4, Canada \\ vlev@cs.ubc.ca \\ 2 UPMC Univ Paris 06, UMR 7222 \\ Institut des Systèmes Intelligents et de Robotique, F-75005, Paris, France \\ vincent.hayward@isir.fr
}

\begin{abstract}
Raised line patterns are used extensively in the design of tactile graphics for persons with visual impairments. A tactile stroke pattern was therefore developed to enable the rendering of vector graphics by lateral skin deformation. The stroke pattern defines a transversal profile and a longitudinal texture which provide tactile feedback while respectively crossing over the stroke and tracing its length. The stroke pattern is demonstrated with the rendering of lines, circles and polygons, and is extensible to other vector graphics primitives such as curves. The parametric nature of the stroke allows the representation of distinctive line types and the online adjustment of line thickness and other parameters according to user preferences and capabilities. The stroke pattern was informally evaluated with four visually impaired volunteers.
\end{abstract}

Key words: assistive technology, visual impairment, tactile graphics, tactile display, haptic rendering, laterotactile rendering

\section{Introduction}

The accessibility of graphical content is gaining in importance for persons with visual impairments as visual representations of information become increasingly ubiquitous in applications ranging from home appliances to computer interfaces. The accessibility of graphics is particularly critical in education where visually impaired students require equal access to visual teaching aids such as technical diagrams $[1,2]$. Tactile graphics, however, are generally produced on physical media such as embossed paper or thermoformed plastic [1] which are cumbersome to produce and distribute, often deteriorate with use, and do not afford access to dynamic content such as interactive geographic maps.

These issues could potentially be addressed through the development of virtual or refreshable tactile graphics interfaces. Force-feedback interfaces have for example been used to allow single-point interaction with virtual environments or surfaces (e.g. [3]). An alternative consists of using a transducer known as a tactile display that produces distributed tactile sensations by deforming or otherwise stimulating the skin [4]. A first class of tactile displays presents a large, 
Proc. of Europhaptics 2010, Part II, Kappers, A.M.L. et al. (Eds.), LNSC 6192, Springer-Verlag, pp. 25-30.

programmable surface to be explored by the fingers or hands, often in the form of an array of actuated pins (e.g. [5]). A second class produces a large virtual surface out of a smaller tactile display by dynamically altering the sensation produced in response to displacements of the device (e.g. [6]).

The work presented in this paper leverages a novel approach to skin stimulation that produces virtual tactile graphics by laterally deforming the fingerpad skin with the Tactograph, a haptic interface that combines a STRess ${ }^{2}$ laterotactile display and an instrumented planar carrier (Fig. 1a-b). The latest STRess ${ }^{2}$ consists of an array of $8 \times 8$ independent piezoelectric actuators forming a dense array of 64 laterally-moving skin contactors within an area of $1 \mathrm{~cm}^{2}$ [7]. The tip of each actuator can be deflected towards the left or right by a maximum of approximately $0.1 \mathrm{~mm}$. Virtual tactile graphics are produced by stimulating the skin with the tactile display as it slides within the carrier's $21 \times 15 \mathrm{~cm}$ workspace.

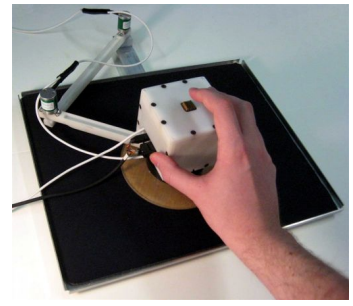

(a)

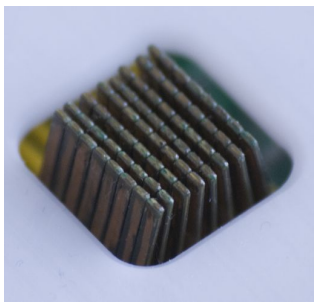

(b)

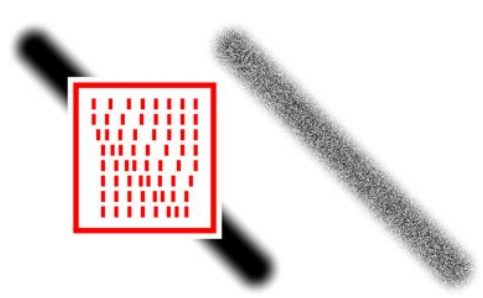

(c)

Fig. 1. Pictures of (a) the Tactograph and (b) its array of skin contactors, and (c) simulation of actuator activation over $5-\mathrm{mm}$ wide stroked lines.

Laterotactile rendering algorithms can be described as sets of deflection functions $\delta_{i, j}(\boldsymbol{P}, \theta, t)$ that define the behaviour of each actuator $i, j$ as a function of time $t$ as well as the position $\boldsymbol{P}$ and orientation $\theta$ of the tactile display. Rendering algorithms can often be simplified such that the deflection $\delta(\boldsymbol{p})$ of an actuator depends solely on its position $\boldsymbol{p}$ within the virtual canvas. Vibration can also be rendered by introducing a time-varying sinusoidal oscillation with variable amplitude and a maximum frequency of $50 \mathrm{~Hz}$. Virtual tactile graphics are illustrated as shown in Fig. 1c by mapping local deflection and vibration to pixel intensity and white noise respectively.

Previous work has demonstrated that complex illustrations, simple shapes and textures can be produced with rendering algorithms that generate localized vibrations, grating patterns and raised dots based on bitmapped modulation masks $[8,9]$. The work presented in this paper introduces vector graphics capabilities to this framework through the development of a tactile stroke pattern that emulates the properties of a raised line. The stroke pattern is defined by a transversal profile which triggers the sensation of crossing over the stroke, and a longitudinal texture which provides feedback as the stroke is traced. The stroke pattern can be applied to a variety of vector graphics primitives and is demon- 
Proc. of Europhaptics 2010, Part II, Kappers, A.M.L. et al. (Eds.), LNSC 6192, Springer-Verlag, pp. 25-30.

strated with the rendering of lines, circles and polygons. Strokes and stroked shapes were informally evaluated with four visually impaired volunteers.

\section{Stroke}

The stroke is similar in concept to a brush stroke or raised line and defines the tactile appearance of a shape's outline. A stroke's rendering is composed of a transversal deflection profile that depends on the distance $r$ across the shape's path as well as a longitudinal texture varying with the distance $l$ along its length.

The transversal profile triggers the sensation of brushing over a raised line when moving across the stroke by causing a sinusoidal swing in actuator deflection. A smooth profile (Fig. 2a) results in a natural sensation which becomes increasingly subtle as the stroke thickness is increased. The sharpness of the stroke is restored by shortening the sinusoidal transition at its edges (Fig. 2b). Sharp edges, however, can create the sensation of touching two distinct lines when separated by a certain distance. This effect can optionally be embraced and reinforced by rendering the stroke as an outline with maximal actuator swing at its edges (Fig. 2c). A transversal texture in the form of a sinusoidal oscillation can finally be superposed over a scaled-down deflection profile (Fig. 2d).

The transversal profile provides only minimal feedback when following the length of a stroke. A longitudinal texture is therefore introduced to provide additional tactile feedback while tracing a shape's contour. The resulting stroke deflection is produced by modulating the stroke profile with a grating waveform varying along the length of the shape's path. The grating texture imitates the sensation of brushing against a corrugated surface by producing smooth swings in actuator deflections that are perceived as raised bumps or ridges [8]. A grating cycle is composed of a sinusoidal swing that creates the sensation of a ridge followed by an optional gap that reinforces the salience of the tactile feature. Although otherwise effective, a dense texture can interfere with the perception

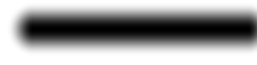

(a)

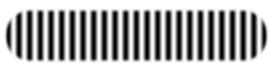

(e)

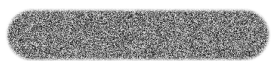

(i)

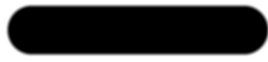

(b)

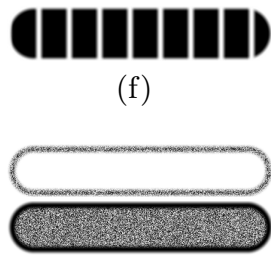

(j)

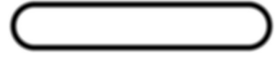

(c)

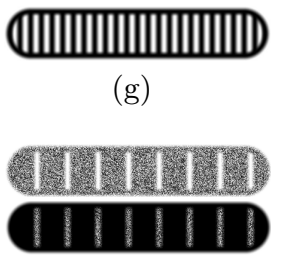

(k)

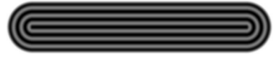

(d)

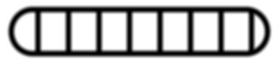

(h)

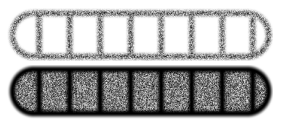

(1)

Fig. 2. Examples of the use of (a-d) transversal profiles, (e-h) longitudinal textures and (i-l) vibrations in the rendering of a stroked line. 
Proc. of Europhaptics 2010, Part II, Kappers, A.M.L. et al. (Eds.), LNSC 6192, Springer-Verlag, pp. 25-30.

of the profile by introducing breaks in the edges (Fig. 2e). The frequency of edge breaks can be reduced through the use of a sparse grating texture (Fig. 2f). As an alternative, an outlined stroke (Fig. 2g-h) can be produced to reinforce the stroke's edges. The salience of the grating texture is dependent on its physical extent and hence affected by the width and sharpness of the stroke profile.

Vibration can also be used to reinforce the intensity of a stroke and to allow it to be felt even in the absence of exploratory movement. Vibration can be applied either to the active or inactive pattern of a stroke (Fig. 2i-l).

\section{Shapes}

Shapes are rendered by applying the stroke pattern according to the definition of a coordinate system that specifies both a transversal distance $r$ from the shape contour and a longitudinal distance $l$ along its length. The concept is described below for lines, circles and polygons but could easily be generalized and adapted to other common vector graphics primitives such as curves, arcs and open paths.

A line is rendered by defining the transversal distance $r$ and the longitudinal distance $l$ respectively as the minimal distance to the line segment and the distance to the actuator's projection along its length. This results in rounded lines caps through which the longitudinal texture extends gracefully (Fig. 3a). A circle is similarly rendered by defining $r$ and $l$ as the minimal distance to its contour and arc length along it respectively (Fig. 3b). Continuity is ensured by fitting a whole number of texture cycles within the circle's circumference.

A polygon is rendered by defining the transversal distance $r$ as the minimal distance to the its contour, which corresponds to the minimum transversal distance to its line segments. The definition of the longitudinal distance $l$ is more ambiguous due to complications at the vertices. The simplest approach consists of extending the longitudinal path to the midline of the joints. This definition, however, causes discontinuities in the rendering at the junction of the line seg-

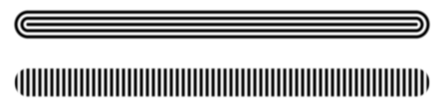

(a)

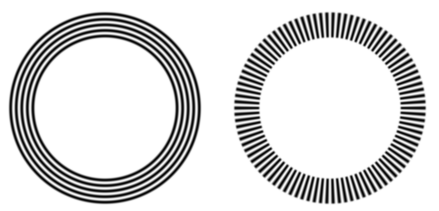

(b)
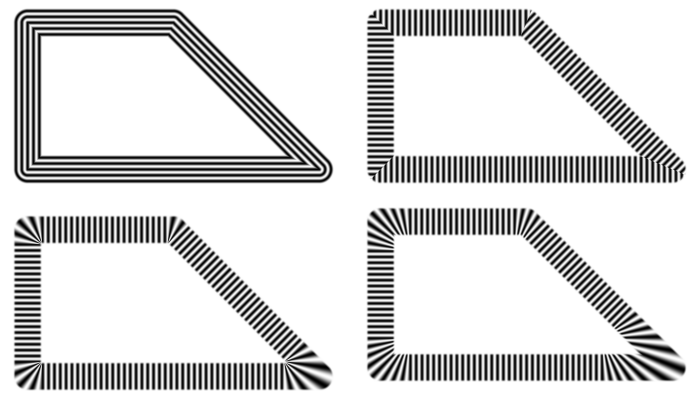

(c)

Fig. 3. Illustration of the transversal and longitudinal coordinate system for stroked (a) lines, (b) circles, and (c) polygons. 


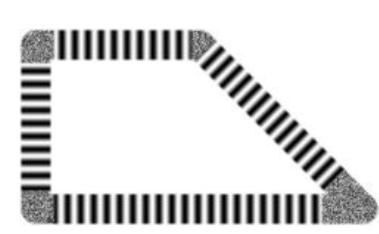

(a)

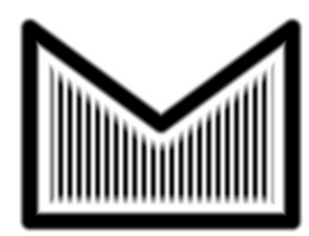

(b)

Fig. 4. Examples of polygons with (a) vertex markers and (b) a fill texture.

ments which can be eliminated by rounding the longitudinal path. A further complication is encountered close to the arc's pivot point where the spatial frequency of the texture increases without bounds. This singularity can be moved out of the stroke by defining the longitudinal path as if for a wider stroke, resulting in the early onset of the rounding near joints. All three approaches are illustrated in Fig. 3c. Texture continuity is enforced such that a whole number of cycles fits within the length of the selected longitudinal path.

Markers can also be superposed on a polygon's vertices to improve their salience, facilitate their localization and hide discontinuities in their rendering (Fig. 4a). Vertex markers have a conic shape that extends from the point of intersection of the joint's edges and are rendered either as plain or vibrating patterns. Their angular coverage can either be fixed or set according to the properties of the joint. A filling texture such as a grating [8] can similarly be applied to the interior of a polygon or circle (Fig. 4b). The texture begins at the inner edge of the stroke and fades in linearly over a small distance to avoid discontinuities, with an optional gap for increased contrast.

\section{Discussion}

The effectiveness of strokes and stroked shapes was informally evaluated by soliciting feedback from four visually-impaired volunteers. The following discussion forms a synthesis of the preliminary insights gained from their comments.

Although individual preferences vary, alteration of the sharpness, thickness and texture of a stroke results in distinctive tactile patterns that can be used to produce contrasting line types. Smooth strokes are noisy when thin and weak when thick, but otherwise usable for a range of approximately 2 to $10 \mathrm{~mm}$. The edges of sharp or outlined strokes, on the other hand, remain strong but are felt as disjoint lines when the thickness exceeds approximately $5 \mathrm{~mm}$. Transversal and longitudinal textures both add substance to the stroke with the latter providing better tracing feedback. A dense grating with a spatial wavelength of $2 \mathrm{~mm}$ is generally preferred and the breaks introduced in the edges do not appear to warrant correction with a sparse grating or a stroke outline. Vibrations result in less pleasant but much more intense sensations than smooth deflections and are therefore ideal to introduce contrast. Vibration, however, may cause tactile adap- 
Proc. of Europhaptics 2010, Part II, Kappers, A.M.L. et al. (Eds.), LNSC 6192, Springer-Verlag, pp. 25-30.

tation with prolonged exposure and tends to overpower nearby non-vibrating patterns. The geometry of stroked shapes can be traced and understood with relative ease. The rounding of the longitudinal path at polygon vertices appears to slightly weaken sharp corners while the discontinuities otherwise introduced have minimal effect. Vibrating markers are effective at highlighting vertices but their exact shape is not perceptible and should be selected to maximize area. Fill textures are effective but sometimes interfere with the shape contour, particularly in the absence of a gap or clear contrast between the stroke and texture.

This informal evaluation suggests that the stroke pattern has great potential for the laterotactile rendering of vector graphics. The results indicate that stroke parameters could be selected not only for improved perceptibility, but also to present contrasting line types. Stroke parameters could moreover be adjusted online so as to adapt to the user's preferences and capabilities. Much work nevertheless remains to formally evaluate the effectiveness of the stroke pattern and the effect of its rendering parameters on the discriminability and identifiability of strokes and stroked shapes. Preliminary results suggest that stroked shapes should match or outperform the dotted and vibrating patterns evaluated in [8]. The rendering of stroke intersections and the achievable density of stroked patterns may also require further investigation for practical applications. This work is a first step towards a complete vector graphics drawing library which could enable the automated adaptation of vector graphics content such as those produced using the increasingly popular Scalable Vector Graphics (SVG) format.

\section{References}

1. Edman, P.K.: Tactile Graphics. AFB Press, New York (1992)

2. Aldrich, F.K., Sheppard, L.: Tactile graphics in school education: perspectives from pupils. British Journal of Visual Impairment 19(2) (2001) 69-73

3. Rassmus-Gröhn, K., Magnusson, C., Eftring, H.: User evaluations of a virtual hapticaudio line drawing prototype. In: Proc. Workshop on Haptic and Audio Interaction Design. (2006)

4. Vidal-Verdú, F., Hafez, M.: Graphical tactile displays for visually-impaired people. IEEE Trans. Neural Syst. Rehabil. Eng 15(1) (2007) 119-130

5. Watanabe, T., Kobayashi, M., Ono, S., Yokoyama, K.: Practical use of interactive tactile graphic display system at a school for the blind. In: Proc. Fourth International Conference on Multimedia and Information and Communication Technologies in Education (m-ICTE). (2006) 1111-1115

6. Jansson, G., Juhasz, I., Cammilton, A.: Reading virtual maps with a haptic mouse: Effects of some modifications of the tactile and audio-tactile information. British Journal of Visual Impairment 24(2) (2006) 60-66

7. Wang, Q., Hayward, V.: Compact, portable, modular, high-performance, distributed tactile display device based on lateral skin deformation. In: Proc. Haptics Symposium. (2006) 67-72

8. Lévesque, V., Hayward, V.: Tactile graphics rendering using three laterotactile drawing primitives. In: Proc. Haptics Symposium. (2008) 429-436

9. Petit, G., Dufresne, A., Lévesque, V., Hayward, V., Trudeau, N.: Refreshable tactile graphics applied to schoolbook illustrations for students with visual impairment. In: Proc. ACM Conference on Computers and Accessibility (ASSETS). (2008) 89-96 